\title{
EFFECTS OF POSTISCHEMIC LEFT VENTRICULAR PRESSURE-VOLUME UNLOADING ON CONTRACTILE RECOVERY AND MYOCARDIAL BLOOD FLOW IN THE REGIONALLY STUNNED CANINE HEART
}

Alfred C. Nicolosi, MD

John G. Markley, MD

Gordon N. Olinger, MD
Objective: Myocardial stunning remains a clinical problem without definitive therapy. This study tested the hypothesis that mechanical therapy with a ventricular assist device would accelerate recovery of contractility in stunned myocardium by increasing the postischemic myocardial blood flow. Methods: Regional stunning was induced in dogs $(25 \mathrm{~kg})$ by 15 minutes of coronary occlusion and 180 minutes of reperfusion. One group (ventricular assist device; $\mathbf{n}=10$ ) was reperfused in conjunction with left ventricular unloading with a centrifugal-pump ventricular assist device. A second group (control; $n=8$ ) underwent unmodified reperfusion. Hemodynamic and regional function data were acquired in all dogs with the heart in the working state before and during ischemia and after 180 minutes of reperfusion. Regional myocardial blood flow was measured at these same intervals and after 30 minutes of reperfusion, at which time the left ventricle was mechanically unloaded in animals with a ventricular assist device. Results: Regional stunning was observed in all animals, but cardiogenic shock developed in none of them. After 180 minutes of reperfusion, animals with a ventricular assist device had greater systolic shortening in the risk segment than did control animals $(11.5 \% \pm 2.8 \%$ vs $1.1 \% \pm 1.3 \% ; P<.05)$ and had no differences in either the slope or $x$-axis intercept of regional preload recruitable stroke work relations compared with preischemic values. Differences in contractile recovery did not correlate, however, with postischemic myocardial blood flow. Hyperperfusion mediated by the ventricular assist device was not observed in either stunned or remote segments. Conclusions: Mechanical left ventricular unloading attenuates regional myocardial stunning within 3 hours in normotensive dogs, independent of effects on myocardial blood flow. The mechanism underlying this effect remains undefined, but these data support expanded use of mechanical therapy for stunned myocardium in clinical settings. ( $\mathrm{J}$ Thorac Cardiovasc Surg 1999;118:181-8)
$\mathrm{M}$ yocardial stunning, the persistent contractile defect associated with ischemia-reperfusion, remains an important problem in cardiac surgery and in acute coro-

\footnotetext{
From the Division of Cardiothoracic Surgery, The Medical College of Wisconsin, Milwaukee, Wis.

Supported in part by educational gifts from Medtronic, Inc, Minneapolis, Minn, and Sarns Inc/3M Health Care, Ann Arbor, Mich.

Received for publication Sept 9, 1998; revisions requested Oct 30, 1998; revisions received March 1, 1999; accepted for publication March 30, 1999.

Address for reprints: Alfred Nicolosi, MD, Division of Cardiothoracic Surgery, Froedtert Memorial Lutheran Hospital, 9200 W Wisconsin Ave, Milwaukee, WI 53226.

Copyright (C) 1999 by Mosby, Inc.

$0022-5223 / 99 \$ 8.00+0 \quad \mathbf{1 2 / 1 / 9 8 9 1 9}$
}

nary syndromes. Specific pharmacologic therapy for stunning is yet undefined because the underlying pathophysiology remains incompletely understood. ${ }^{1,2}$

Mechanical therapy with a ventricular assist device (VAD) for pressure-volume unloading has previously been shown to reduce infarct size ${ }^{3-6}$ and to improve survival from otherwise lethal cardiac failure, ${ }^{7-11}$ but neither infarction nor cardiogenic shock (which results in sustained myocardial hypoperfusion) reflects the known pathophysiology of stunning. ${ }^{1,12}$ A direct effect of pressure-volume unloading on recovery of truly stunned myocardium has not been well established, and data showing that postcardiotomy VAD support is often needed for up to 10 days ${ }^{13}$ suggest, in fact, that mechanical therapy does not accelerate recovery of stunned myocardium. 
We hypothesized that postischemic pressure-volume unloading would accelerate contractile recovery of truly stunned myocardium and would do so through an effect on postischemic myocardial blood flow. We reasoned that decreased wall tension in the unloaded ventricle would result in a substantial increase in myocardial blood flow, particularly during systole. Although stunning is not associated with persistent, postischemic hypoperfusion, we believed that hyperperfusion might accelerate energy-dependent cellular repair processes.

\section{Methods}

Instrumentation. Mongrel dogs $(\mathrm{n}=18$; approximately 25 $\mathrm{kg}$ ), randomly assigned to control or VAD groups, were anesthetized with intravenous pentobarbital $(200 \mathrm{mg} / \mathrm{kg})$ and barbital $(26 \mathrm{mg} / \mathrm{kg})$, intubated, and their lungs were ventilated with supplemental oxygen. Normothermia was maintained with heating blankets. Catheters were placed into the femoral artery to measure blood pressure and into the femoral vein for infusion of drugs and crystalloid solution. The heart was exposed by a left thoracotomy and the left anterior descending coronary artery (LAD) was encircled with a silk thread immediately distal to the first diagonal branch. A micromanometer-tipped catheter (Millar Instruments, Inc, Houston, Tex) was inserted into the subclavian artery and advanced retrogradely across the aortic valve to measure left ventricular (LV) pressure. Two cylindrical, ultrasonic dimension crystals (Triton Technology, Inc, San Diego, Calif) were embedded in the anterior LV free wall, approximately $1 \mathrm{~cm}$ apart, to measure instantaneous free wall segment length in the distal LAD territory (stunned segment). A second crystal pair was embedded in the lateral LV free wall to measure free wall segment length in the circumflex territory (remote segment). The crystals were connected to an ultrasonic dimension system (Crystal Biotech, Hopkinton, Mass). An ultrasonic flow probe (Transonics Systems, Inc, Ithaca, NY) was placed around the pulmonary artery and connected to a flowmeter (model T101; Transonics) to measure cardiac output. A coronary flow probe $(1.5 \mathrm{~mm}$; Transonics) was placed on the LAD immediately distal to the silk snare in several animals to measure instantaneous blood flow. A catheter was inserted into the left atrium to measure pressure and to infuse microspheres for measurement of myocardial blood flow. A snare was placed around the inferior vena cava (IVC) to intermittently vary preload.

Animals were heparinized (100 units of heparin per kilogram, given intravenously) and cannulated for LV assist. A 34F drainage cannula (Research Medical, Inc, Salt Lake City, Utah) was inserted into the left atrium and advanced across the mitral valve into the left ventricle. A $6.5-\mathrm{mm}$ inflow cannula (Sarns Inc/3M Health Care, Ann Arbor, Mich) was inserted into the mid-descending thoracic aorta. Both cannulas were de-aired and connected to a nonpulsatile centrifugalpump VAD (Medtronic Bio-Medicus, Eden Prairie, Minn) that was primed with approximately $200 \mathrm{~mL}$ of saline solution. Hematocrit value and arterial blood gases were monitored throughout each experiment. Supplemental barbiturate anesthesia was given as indicated by corneal and hemodynamic reflexes.

Protocol. The condition of the animals was allowed to stabilize after instrumentation. The LAD was then occluded by snaring the silk suture. Lidocaine $(30 \mathrm{mg}$ ) was given intravenously before ischemia and $100 \mathrm{mg}$ was given during the ischemic period. Ventricular fibrillation was treated as necessary with direct-current countershock. The LAD snare was released after 15 minutes and the segment was reperfused for 180 minutes. One group (VAD; $\mathrm{n}=10$ ) was treated immediately on release of the snare with LV pressure-volume unloading. VAD flows in these animals were adjusted and/or crystalloid solution was given as needed to maintain both cardiac output and mean arterial pressure at preischemic values and to maintain maximal LV unloading (defined by absence of ventricular ejection on the arterial waveform and by a peak $\mathrm{LV}$ pressure $<50 \mathrm{~mm} \mathrm{Hg}$ ). VAD flows varied, therefore, with the individual animal's baseline cardiac output but were usually maintained at 2 to $3 \mathrm{~L} / \mathrm{min}$. VAD support was terminated after 165 minutes of reperfusion, and the condition of the animals was allowed to stabilize with the heart in the working mode for 15 minutes. A second group of animals (control; $n$ $=8$ ) underwent unmodified reperfusion for 180 minutes.

All animals were put to death at the end of the protocol by inducing ventricular fibrillation in the presence of deep general anesthesia. The heart was excised and immersed in formalin. All animals received humane care in compliance with the "Principles of Laboratory Animal Care" formulated by the National Society for Animal Research and the "Guide for the Care and Use of Laboratory Animals" prepared by the National Academy of Sciences and published by the National Institutes of Health (DHEW [NIH] Publication No. 85-23, revised 1985). This study was approved by the Medical College of Wisconsin Animal Care Committee.

Data analysis. Hemodynamic and regional dimension data were collected at baseline, after 10 minutes of ischemia, and after 180 minutes of reperfusion with the heart in the working state in all animals. Data were digitized at $250 \mathrm{~Hz}$ per channel and stored directly to computer disk with commercial software (Codas, Akron, Ohio). Heart rate, mean arterial pressure, and left atrial pressure were measured in triplicate during a steady state and averaged. Right ventricular stroke volume was determined by integrating pulmonary artery flow on a beat-to-beat basis and was assumed to be equal to LV stroke volume. Cardiac output was calculated as the product of stroke volume and heart rate. The first derivative of LV pressure with respect to time $(\mathrm{dP} / \mathrm{dt})$ was used to define events in the cardiac cycle.

Steady-state systolic shortening (SS; percent) was defined by the formula:

$$
\mathrm{SS}=(\mathrm{EDL}-\mathrm{ESL}) / \mathrm{EDL}
$$

where EDL is end-diastolic length and ESL is end-systolic length. End-diastole was defined as the point $40 \mathrm{~ms}$ before peak positive $\mathrm{dP} / \mathrm{dt}$ and end-systole as the point $12 \mathrm{~ms}$ before peak negative $\mathrm{dP} / \mathrm{dt}$.

Instantaneous pressure-dimension relations were analyzed with commercial software (Mathcad 6.0 Standard Edition; Mathsoft Inc, Cambridge, Mass). Contractility was defined 
by the regional preload recruitable stroke work (PRSW) relation as described by Glower and associates ${ }^{14}$ and modified by us. ${ }^{15,16}$ In brief, a family of pressure-dimension loops was generated for each region using transient IVC occlusion to vary preload. Regional stroke work $(\mathrm{SW} ; \mathrm{mm} \mathrm{Hg} \cdot \mathrm{mm})$ was defined as:

$$
\mathrm{SW}=\int \mathrm{LV} \text { pressure } \cdot \Delta \mathrm{d}
$$

where LV pressure is integrated over the period from enddiastole to end-systole and $\Delta \mathrm{d}=(\mathrm{EDL}-\mathrm{ESL})$. Stroke work was then plotted on a beat-to-beat basis as a function of enddiastolic length and fitted to the linear formula:

$$
\mathrm{SW}=\mathrm{M}_{\mathrm{w}}\left(\mathrm{EDL}-\mathrm{D}_{\mathrm{w}}\right)
$$

where $\mathrm{D}_{\mathrm{W}}$ is the dimension-axis intercept, and the slope $\left(\mathrm{M}_{\mathrm{w}}\right.$; $\mathrm{mm} \mathrm{Hg}$ ) of the relation varies directly with the contractile state in a load-independent fashion.

Regional myocardial blood flow was determined in 6 control animals and 7 VAD animals with the use of radioactive microspheres at baseline, after 10 minutes of ischemia, and after both 30 and 180 minutes of reperfusion. Preliminary assessment of instantaneous LAD blood flow with the coronary flow probe demonstrated a marked hyperemic response immediately on reperfusion that gradually returned to a stable level within 30 minutes in both groups. Accordingly, microspheres were injected after 30 minutes of reperfusion, while VAD animals were being assisted, to assess for differences in regional myocardial blood flow caused by LV unloading. Approximately 1 to 2 million microspheres (16 $\mu \mathrm{m}$ diameter; $20 \mu \mathrm{Cu}$ dose) were injected into the left atrium after mechanical agitation, with a different isotope label being used for each injection. A reference sample of blood was withdrawn from the femoral artery at a constant rate of $7.5 \mathrm{~mL} / \mathrm{min}$, beginning 15 seconds before injection and continuing for 120 seconds. The heart was excised at the end of the experiment and fixed in formalin for 48 hours. Transmural samples of both ischemic and remote regions were divided into epicardial and endocardial halves and then divided in halves again to separate out the mid-myocardial zones. Samples were weighed and counted for radioactivity (Beckman gamma counter; Beckman Instruments, Inc, Fullerton, Calif) with the reference blood samples. Blood flow was calculated from radioactivity counts in milliliters per minute per gram of tissue.

Statistical analysis. Differences that occurred over time within groups were assessed by analysis of variance for repeated measures followed by the Student-Newman-Keuls test where significant differences were identified. The unpaired Student $t$ test was used to compare differences between control and VAD groups. Paired $t$ tests were used to compare differences between stunned and remote regions in the same animal. All data are presented as mean \pm standard error of the mean (SEM).

\section{Results}

Steady-state pressure-dimension data from representative animals are shown in Fig 1. Regional ischemia is associated with marked diastolic lengthening and para- doxic systolic bulging (stunned segment, both animals) but has minimal effect on either remote segment function or aortic pressure. Mechanical unloading (VAD animal, 30 minutes of reperfusion) eliminates aortic pulsatility and markedly decreases peak systolic LV pressure. Unloading also results in marked decreases in the end-diastolic length of both remote and stunned segments and minimizes systolic excursions. Diastolic lengthening and systolic paradox persist throughout unmodified reperfusion (control animal, 180 minutes of reperfusion), but there is restoration to near-normal end-diastolic length and systolic function in the VAD animal at 180 minutes (after weaning from the device).

Mean steady-state hemodynamic data are summarized in Table I. Regional ischemia resulted in increased left atrial pressure in both groups but was not associated with any changes in heart rate, mean arterial pressure, or stroke volume. The ischemia-induced increase in left atrial pressure was no longer observed after 180 minutes of unmodified reperfusion (controls) but persisted in VAD animals. A decrease in heart rate was also observed during reperfusion in VAD animals, but stroke volume and mean arterial pressure were unchanged. Average mean arterial pressure during assisted reperfusion was $91 \pm 6 \mathrm{~mm} \mathrm{Hg}$, which did not differ from pressure in control animals during reperfusion.

Mean data for regional systolic shortening are summarized in Fig 2. Preischemic shortening was less in remote than in stunned segments of both groups, probably as a result of remote crystal placement closer to the base of the heart. Ischemia resulted in similar degrees of systolic paradox in the 2 groups (Fig 2, A), with recovery after 180 minutes of reperfusion that was greater in VAD animals than in control animals $(P<$ $.05)$, but which remained less than preischemic values in both groups. Small but unimportant differences in remote segment shortening (Fig 2,B) were noted in both groups. Table II shows mean results for regional pressure-dimension analyses. Ischemia was associated with negative stroke work values for all or most beats during IVC occlusions in both groups because of systolic paradoxic wall motion (ie, $\Delta \mathrm{d}<0$ ). The corresponding values for the slope regression $\left(\mathrm{M}_{\mathrm{w}}\right)$, although often greater than zero, are not believed to reflect what would appear to be complete loss of contractile function and are thus omitted. The value for mean slope at 180 minutes of reperfusion is also omitted for control animals, because only 1 animal had positive values for stroke work during IVC occlusion. All VAD animals, on the other hand, had positive stroke work values after 180 minutes, which allowed comparison of slope regression $\left(\mathrm{M}_{\mathrm{w}}\right)$ with preischemic values 


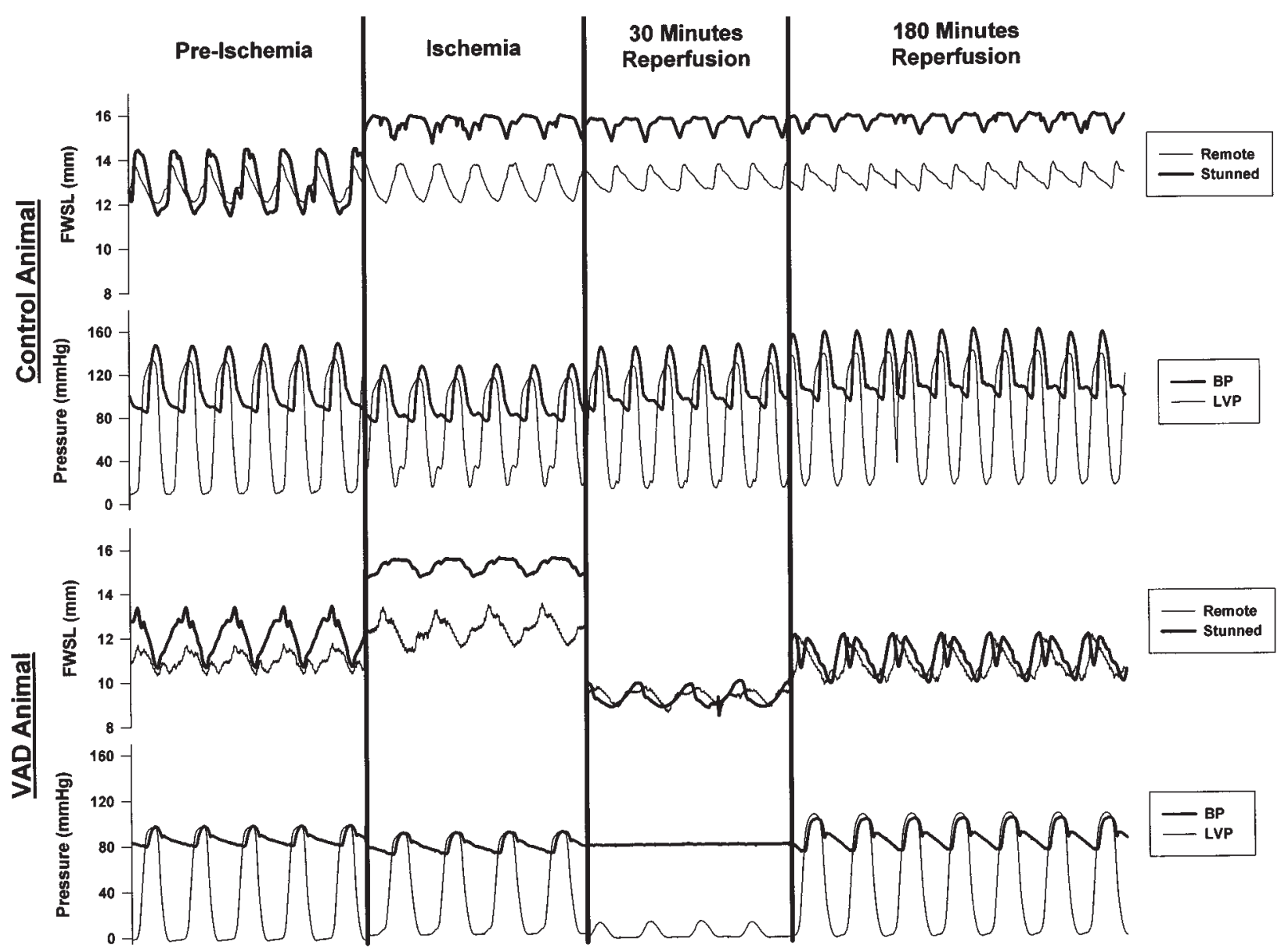

Fig 1. Representative pressure and regional dimension data from regionally stunned dogs subject to either unmodified reperfusion (Control Animal) or postischemic left ventricular pressure-volume unloading (VAD Animal). Stunned $=$ Stunned segment dimension; remote $=$ remote segment dimension; $B P$, aortic pressure; $L V P$, left ventricular pressure; $F W S L$, free wall segment length.

and demonstrated complete recovery of contractility. The $\mathrm{x}$-axis intercept $\left(\mathrm{D}_{\mathrm{w}}\right)$, which reflects unstressed segment length, was higher than preischemic values after 180 minutes of reperfusion in control animals but was unchanged in VAD animals. No differences were observed in slope $\left(M_{w}\right)$ or $x$-axis intercept $\left(D_{w}\right)$ in the remote segments of either group.

Mean data for regional myocardial blood flow are summarized in Table III. Values for endocardial and epicardial myocardial blood flow tended to differ only slightly, with nearly identical patterns of change over time. Ischemia was associated with marked decreases in myocardial blood flow in the stunned segments in both groups. Stunned-segment endocardial blood flow tended to be increased in VAD animals after 30 minutes of reperfusion (during ventricular unloading) but was not statistically different from flow in either preis- chemic animals or control animals. Remote-segment myocardial blood flow was unchanged by LV unloading. After 180 minutes of reperfusion (VAD animals weaned from the device), regional blood flows were unchanged from preischemic values in both groups.

\section{Discussion}

This study demonstrates that mechanical pressurevolume unloading initiated immediately on reperfusion directly enhances recovery of contractile function in regionally stunned myocardium. Animals treated with a VAD had greater recovery of regional systolic shortening after 180 minutes of reperfusion than did unassisted controls, and they had normal segmental contractility on the basis of regional PRSW relations $\left(\mathrm{M}_{\mathrm{w}}\right.$ and $\left.\mathrm{D}_{\mathrm{w}}\right)$.

The mechanism by which unloading accelerates contractile recovery does not appear to result from associat- 
Table I. Mean ( \pm SEM) hemodynamic data in regionally stunned dogs undergoing unmodified (control; $n=8$ ) or assisted (VAD; $n=10)$ reperfusion

\begin{tabular}{lccc}
\hline & $\begin{array}{c}\text { Before } \\
\text { ischemia }\end{array}$ & Ischemia & $\begin{array}{c}\text { Reperfusion } \\
(180 \mathrm{~min})\end{array}$ \\
\hline $\begin{array}{l}\text { Heart rate (beats/min) } \\
\quad \text { Control }\end{array}$ & $123 \pm 8$ & $118 \pm 8$ & $116 \pm 9$ \\
$\quad$ VAD & $120 \pm 4$ & $120 \pm 5$ & $100 \pm 8^{*}$ \\
$\begin{array}{l}\text { Mean arterial pressure } \\
\quad \text { (mm Hg) }\end{array}$ & & & \\
$\quad$ Control & $106 \pm 7$ & $100 \pm 6$ & $109 \pm 10$ \\
$\quad$ VAD & $107 \pm 7$ & $100 \pm 7$ & $98 \pm 7$ \\
Stroke volume (mL) & & & \\
$\quad$ Control & $21.4 \pm 2.2$ & $22.6 \pm 2.0$ & $16.7 \pm 2.2$ \\
$\quad$ VAD & $18.1 \pm 2.2$ & $18.3 \pm 1.4$ & $18.4 \pm 1.6$ \\
Left atrial pressure & & & \\
$\quad(\mathrm{mm} H \mathrm{Hg})$ & & & \\
Control & $6.7 \pm 0.9$ & $11.6 \pm 1.1^{*}$ & $7.4 \pm 1.0$ \\
$\quad$ VAD & $8.1 \pm 1.8$ & $12.1 \pm 2.5^{*}$ & $12.9 \pm 2.7^{*}$ \\
\hline
\end{tabular}

$* P<.05$ versus before ischemia.

ed effects on myocardial blood flow. We had hypothesized that VAD therapy would accelerate recovery of contractile function by increasing postischemic myocardial blood flow beyond normal levels and would thus improve oxygen delivery to energy-depleted areas. VAD therapy has previously been demonstrated to increase myocardial blood flow more than 2-fold in normal dogs, ${ }^{17}$ perhaps by reducing systolic wall tension, ${ }^{18,19}$ but the present study failed to demonstrate VAD-mediated hyperperfusion of either stunned or normal (remote) myocardium. Wei and Kusagawa ${ }^{20}$ have suggested that autoregulatory mechanisms, which match myocardial blood flow to oxygen demand, may prevent hyperperfusion with unloading, although the integrity of these mechanisms in stunned myocardium may be lost. Using a coronary flow probe, we observed persistent systolic reduction of instantaneous epicardial flow to near zero, despite maximal ventricular unloading. This observation may better explain the absence of VAD-mediated myocardial hyperperfusion, which we postulated would result mainly from increased systolic flow. The effect of unloading on the net oxygen supply/demand ratio, even in the absence of hyperperfusion, might still be expected to accelerate recovery of stunned myocardium by reducing oxygen demand, although recent data indicate that contractile function and oxygen use are uncoupled in stunned myocardium. ${ }^{12,21,22}$

Plasma catecholamine levels were not measured in this study, but the absence of hypertension and the decrease in heart rate in VAD animals (Table I) argue against an increase in catecholamine tone as a
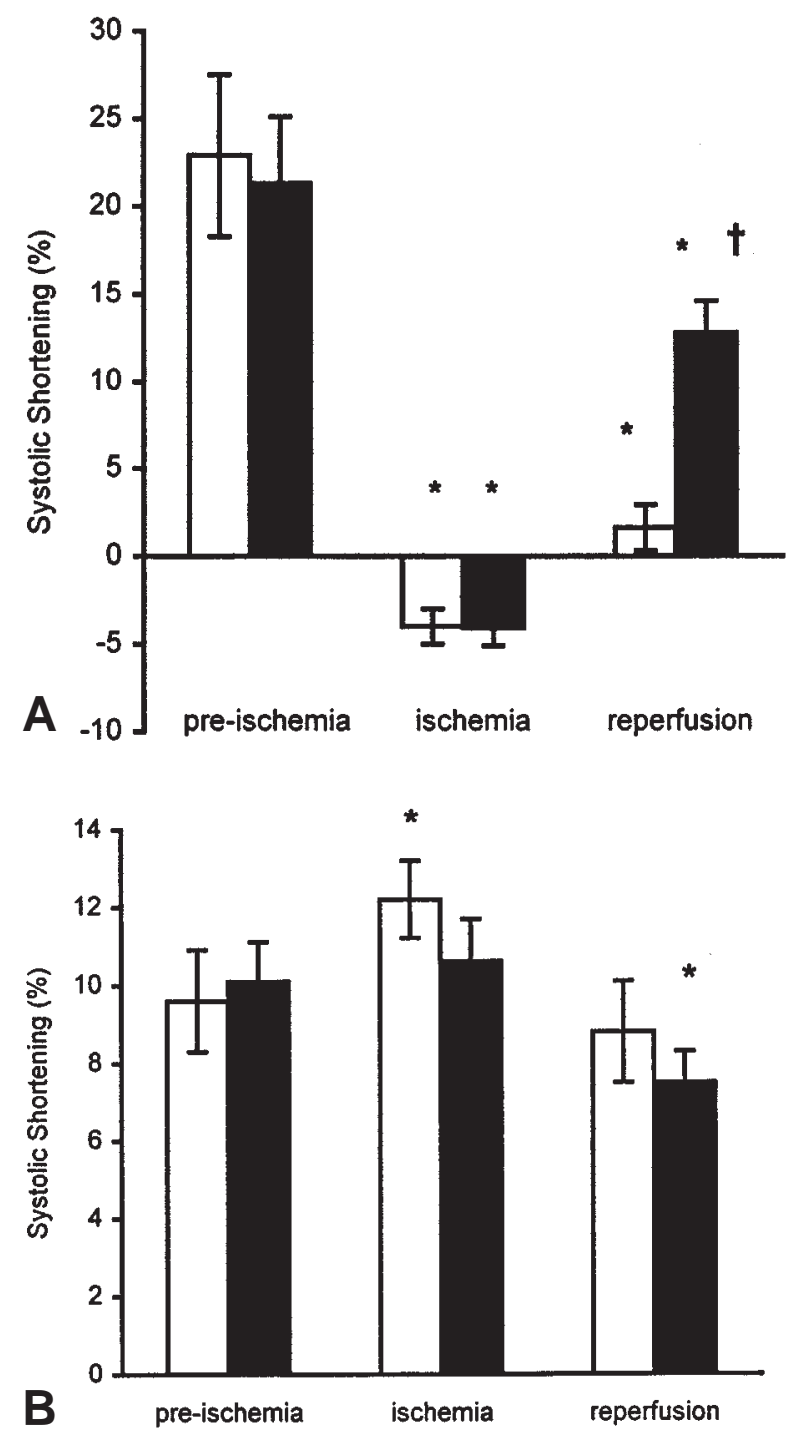

Fig 2. Mean results ( \pm SEM) for regional systolic shortening in stunned (A) and remote (B) segments of regionally stunned dogs undergoing unmodified (controls $[\mathrm{n}=8]$; white bars) or assisted (VAD animals $[\mathrm{n}=10]$; black bars $]$ reperfusion. Preischemic shortening is greater in stunned segments in both groups $(P<.05)$, probably because of crystal placement at different apex-to-base levels between segments. $* P<.05$ versus preischemic values; $\uparrow P<0.05$ versus control animals.

mechanism for enhanced contractile recovery in VAD animals.

The present data also suggest several interesting effects of VAD therapy on passive diastolic properties. Glower and colleagues ${ }^{23}$ have previously demonstrated that reversal of diastolic creep, defined as an increase in unstressed fiber length, parallels recovery of systolic function in stunned myocardium. The $\mathrm{x}$-axis intercept 
Table II. Mean ( \pm SEM) slopes $\left(M_{w}\right)$ and $x$-intercepts $\left(D_{w}\right)$ of regional PRSW analyses from regionally stunned dogs undergoing unmodified (control; $n=8$ ) or assisted (VAD; $n=10$ ) reperfusion

\begin{tabular}{|c|c|c|c|c|}
\hline & \multicolumn{2}{|c|}{$M_{w}$} & \multicolumn{2}{|c|}{$D_{w}$} \\
\hline & Control & $V A D$ & Control & $V A D$ \\
\hline \multicolumn{5}{|l|}{ Stunned segment } \\
\hline Before ischemia & $16.9 \pm 3.2$ & $18.5 \pm 2.0$ & $9.25 \pm 1.1$ & $9.83 \pm 1.09$ \\
\hline Ischemia & NA & NA & $16.00 \pm 0.80^{*}$ & $24.26 \pm 7.51$ \\
\hline Reperfusion & NA & $25.1 \pm 3.8$ & $13.95 \pm 0.69 *$ & $11.05 \pm 1.09$ \\
\hline \multicolumn{5}{|l|}{ Remote segment } \\
\hline Before ischemia & $20.3 \pm 3.8$ & $18.6 \pm 2.1$ & $12.17 \pm 0.75$ & $11.88 \pm 1.00$ \\
\hline Ischemia & $19.2 \pm 1.9$ & $18.1 \pm 2.3$ & $12.34 \pm 0.75$ & $12.17 \pm 1.01$ \\
\hline Reperfusion & $17.4 \pm 2.6$ & $16.4 \pm 2.1$ & $12.21 \pm 0.83$ & $12.08 \pm 1.22$ \\
\hline
\end{tabular}

$N A$, Analysis not appropriate.

$* P<.05$ versus preischemia.

Table III. Mean ( \pm SEM) endocardial and epicardial blood flows ( $\mathrm{mL} / \mathrm{min}$ per gram) in regionally stunned dogs undergoing unmodified (control; $n=6$ ) or assisted (VAD; $n=7$ ) reperfusion

\begin{tabular}{|c|c|c|c|c|}
\hline & Before ischemia & Ischemia & Reperfusion (30 min) & Reperfusion (180 min) \\
\hline \multicolumn{5}{|c|}{ Endocardial blood flow } \\
\hline \multicolumn{5}{|c|}{ Stunned segment } \\
\hline Control & $0.76 \pm 0.19$ & $0.07 \pm 0.04 *$ & $0.63 \pm 0.16$ & $0.83 \pm 0.21$ \\
\hline VAD & $0.85 \pm 0.11$ & $0.05 \pm 0.02 *$ & $1.41 \pm 0.40$ & $0.91 \pm 0.15$ \\
\hline \multicolumn{5}{|c|}{ Remote segment } \\
\hline Control & $0.92 \pm 0.19$ & $1.13 \pm 0.26^{\dagger}$ & $0.93 \pm 0.11$ & $1.37 \pm 0.34$ \\
\hline VAD & $0.89 \pm 0.11$ & $1.23 \pm 0.31^{\dagger}$ & $0.99 \pm 0.23$ & $1.17 \pm 0.15$ \\
\hline \multicolumn{5}{|c|}{ Epicardial blood flow } \\
\hline \multicolumn{5}{|c|}{ Stunned segment } \\
\hline Control & $0.90 \pm 0.21$ & $0.10 \pm 0.02$ & $0.80 \pm 0.11$ & $1.08 \pm 0.18$ \\
\hline VAD & $0.91 \pm 0.13$ & $0.12 \pm 0.03 *$ & $1.03 \pm 0.16$ & $1.18 \pm 0.25$ \\
\hline \multicolumn{5}{|c|}{ Remote segment } \\
\hline Control & $0.74 \pm 0.18$ & $1.42 \pm 0.44^{\dagger}$ & $0.77 \pm 0.12$ & $0.90 \pm 0.20$ \\
\hline VAD & $0.79 \pm 0.12$ & $1.26 \pm 0.51^{\dagger}$ & $0.89 \pm 0.15$ & $1.20 \pm 0.28$ \\
\hline
\end{tabular}

$* P<.05$ versus before ischemia.

${ }^{\dagger} P<.05$ versus stunned segment.

$\left(D_{w}\right)$, which represents unstressed fiber length in the regional PRSW relation, remained increased after 180 minutes of reperfusion in control animals in our study (Table II), indicating persistent creep. Mean x-axis intercept $\left(D_{w}\right)$ for VAD animals after 180 minutes, however, did not differ from preischemic values, indicating resolution of creep and suggesting a potential mechanism for the effect of mechanical unloading on recovery of contractile function. The persistent increase of left atrial pressure in VAD animals after 180 minutes of reperfusion (Table I) is also interesting, particularly in the absence of increased end-diastolic segment lengths in either stunned or remote segments. The divergence of these diastolic pressure and dimension data may be explained by an increase in LV stiffness, which has previously been recognized by Komeda and associates $^{24}$ and which may result from myocardial edema. ${ }^{25,26}$ Further investigation into the effects of LV pressure-volume unloading on diastolic properties is warranted, because they may have important implications for management of patients receiving mechanical support.

Grundeman and coworkers ${ }^{27}$ previously demonstrated $94 \%$ recovery of systolic shortening in regionally stunned myocardium with 6 hours of postischemic VAD therapy. We have demonstrated herein that 3 hours of VAD therapy results in 64\% recovery of systolic shortening, although regional PRSW slope, a more sensitive index of contractility, recovered to $100 \%$ of preischemic function. The relationship between duration of postischemic unloading and the extent of contractile recovery in stunned myocardium needs to be further elucidated, because it too would be an important factor in clinical applications. It must also 
be determined whether delayed unloading after reperfusion would prolong the duration of support needed to effect complete contractile recovery and to define the limits of such a delay.

The model of regional stunning used in this study is important for the absence of cardiogenic shock. Cardiogenic shock results in persistent myocardial hypoperfusion and is thus not consistent with the pathophysiology of stunning. ${ }^{1,12}$ Improved survival and functional recovery observed with VAD therapy in many previous studies ${ }^{7-11}$ most likely result from prevention of ongoing ischemic injury and not from accelerated recovery of truly stunned myocardium. The dog is an accepted model of myocardial stunning, despite the presence of a large coronary collateral system, because stunning tends to be more severe in dogs than in either pigs or baboons, both of which have less well developed coronary collateral systems. ${ }^{28}$ Our data confirm that myocardial blood flow decreases to near zero during acute coronary occlusion in the dog and that postischemic contractile function remains markedly depressed for up to 3 hours, despite restoration of normal myocardial blood flow. We did not perform histologic or special staining tests to look for infarction in this model, because others have previously demonstrated that a 15-minute coronary occlusion is not associated with myocardial necrosis in dogs. ${ }^{29}$

In summary, postischemic LV pressure-volume unloading accelerates recovery of regionally stunned myocardium within 3 hours. The effect of unloading on systolic recovery appears to be independent of effects on systemic hemodynamics and does not correlate with changes in myocardial blood flow. Although the mechanisms underlying this observation remain unexplained, the current results support expanded use of mechanical therapy for myocardial stunning. Further investigation is needed to define involved mechanisms and to refine strategies for the treatment of clinical myocardial ischemia-reperfusion.

We thank Michael Cristoforo, CCP, David Koerten, CCP, Duane Nelson, CCP, and Steven Schiro, CCP, for their invaluable technical support.

\section{REFERENCES}

1. Kloner RA, Bolli R, Marban E, Reinlib L, Braunwald E. Medical and cellular implications of stunning, hibernation and preconditioning: an NHLBI workshop. Circulation 1998;97:1848-67.

2. Hearse DJ, Shattock MJ, Ovize M, Opie LH, Bolli R, Heusch G, et al. Meeting report. Basic Res Cardiol 1998;93:143-72.

3. Wei C-M, Yada I, Kusagawa M. The effect of left ventricular assistance on the area of infarcted myocardium. Trans Am Soc Artif Intern Organs 1986;32:217-20.

4. Allen BS, Okamoto F, Buckberg GD, Bugyi H, Leaf J. Studies of controlled reperfusion after ischemia. XIII. Reperfusion conditions: critical importance of total ventricular decompression during regional reperfusion. J Thorac Cardiovasc Surg 1986;92:605-12.

5. Fonger JD, Zhou Y, Matsuura H, Aldea GS, Shemin RJ. Enhanced preservation of acutely ischemic myocardium with transseptal left ventricular assist. Ann Thorac Surg 1994;57:570-5.

6. Ganim M, Lachterman B, Felli P, Amirian J, Feld S, Vaughn W, et al. Transvalvular left ventricular assistance immediately prior to and throughout reperfusion after a two hour coronary occlusion reduces infarct size (abstract). Circulation 1995;92(Suppl): I458.

7. Mickleborough LL, Rebeyka I, Wilson GJ, Gray G, Desrosiers A. Comparison of left ventricular assist and intra-aortic balloon counterpulsation during early reperfusion after ischemic arrest of the heart. J Thorac Cardiovasc Surg 1987;93:597-608.

8. Magovern GJ Jr, Christlieb IY, Kao RL, Liebler GA, Park SB, Burkholder JA, et al. Recovery of the failing canine heart with biventricular support in a previously fatal experimental model. J Thorac Cardiovasc Surg 1987;94:656-63.

9. Pennington DG, Kanter KR, McBride LR, Kaiser GC, Barner HB, Miller LW, et al. Seven years' experience with the PierceDonachy ventricular assist device. J Thorac Cardiovasc Surg 1988;96:901-11.

10. Drinkwater DC, Laks H. Clinical experience with centrifugal pump ventricular support at UCLA Medical Center. Trans Am Soc Artif Intern Organs 1988;34:505-8.

11. Smalling RW, Sweeney M, Lachterman B, Hess MJ, Morris R, Anderson HV, et al. Transvalvular left ventricular assistance in cardiogenic shock secondary to acute myocardial infarction: evidence for recovery from near fatal myocardial stunning. J Am Coll Cardiol 1994;23:637-44.

12. Stahl LD, Weiss HR, Becker LC. Myocardial oxygen consumption, oxygen supply/demand heterogeneity, and microvascular patency in regionally stunned myocardium. Circulation 1988;77: 865-72.

13. Jett GK. Postcardiotomy support with ventricular assist devices: selection of recipients. Semin Thorac Cardiovasc Surg 1994;6: 136-9.

14. Glower DD, Spratt JA, Snow ND, Kabas JS, Davis JW, Olsen $\mathrm{CO}$, et al. Linearity of the Frank-Starling relationship in the intact heart: the concept of preload recruitable stroke work. Circulation 1985;71:994-1009.

15. Nicolosi AC, Weng Z-C, Detwiler PW, Spotnitz HM. Experimental left ventricular aneurysm and aneurysm repair in swine. J Thorac Cardiovasc Surg 1990;100:745-55.

16. Markley JG, Nicolosi AC. Effects of left heart assist on geometry and function of the interventricular septum. Ann Thorac Surg 1996;62:1752-8

17. Sukehiro S, Flameng W. Effects of left ventricular assist for cardiogenic shock on cardiac function and organ blood flow distribution. Ann Thorac Surg 1990;50:374-83.

18. Rose EA, Marrin CAS, Bregman D, Spotnitz HM. Left ventricular mechanics of counterpulsation and left heart bypass, individually and in combination. J Thorac Cardiovasc Surg 1979;77; 127-37.

19. Ratcliffe MB, Bavaria JE, Wenger RK, Bogen DK, Edmunds LH Jr. Left ventricular mechanics of ejecting, postischemic hearts during left ventricular circulatory assistance. J Thorac Cardiovasc Surg 1991;101:245-55.

20. Wei C-M, Kusagawa M. The effect of left ventricular assistance 
on the area of infarcted myocardium. Trans Am Soc Artif Intern Organs 1986;32:217-20.

21. Furukawa S, Kreiner G, Bavaria JE, Streicher JT, Edmunds LH Jr. Recovery of oxygen utilization efficiency after global myocardial ischemia. Ann Thorac Surg 1991;52:1063-8.

22. Liu B, el Alaoui-Talibi Z, Clanachan AS, Schulz R, Lopaschuk GD. Uncoupling of contractile function from mitochondrial TCA cycle activity and $\mathrm{MV}_{\mathrm{O} 2}$ during reperfusion of ischemic hearts. Am J Physiol 1996;270:H72-80.

23. Glower DD, Schaper J, Kabas JS, Hoffmeister HM, Schaper W, Spratt JA, et al. Relation between reversal of diastolic creep and recovery of systolic function after ischemic myocardial injury in conscious dogs. Circ Res 1987;60:850-60.

24. Komeda M, DeAnda A Jr, Glasson JR, Bolger AF, Daughters GT II, Ingels NB, et al. Complete unloading alone may not adequately protect the left ventricle. Ann Thorac Surg 1997;64:1250-5.

25. Weng Z-C, Nicolosi AC, Detwiler PW, Schierman SW, Goldstein
AW, Spotnitz HM. Effects of crystalloid, blood and UW coronary perfusion on weight, myocardial edema and left ventricular compliance of isolated porcine hearts. J Thorac Cardiovasc Surg 1992;101:504-13.

26. Detwiler PW, Nicolosi AC, Weng Z-C, Spotnitz HM. Effects of perfusion-induced edema on diastolic stress-strain relations in intact swine papillary muscle. J Thorac Cardiovasc Surg 1994;108:467-76.

27. Grundeman PF, Tukkie R, Moulijn AC, Rudolphy VJ, de Jong JW, Frederiks WM. Short-term biventricular circulatory support in the regionally stunned pig myocardium. Thorac Cardiovasc Surg 1993;41:290-6.

28. Shen Y-T, Vatner SF. Differences in myocardial stunning following coronary artery occlusion in conscious dogs, pigs, and baboons. Am J Physiol 1996;270:H312-22.

29. Bolli R, Zhu W-X, Thornby JI, O’Neill PG, Roberts R. Time course and determinants of recovery of function after reversible ischemia in conscious dogs. Am J Physiol 1988;254:H102-14.

\section{Targeted}

The Journal of Thoracic and Cardiovascular Surgery gives you two tables of contents.

The condensed table of contents tells you at a glance what topics and authors are presented each month. The expanded table of contents gives you a brief abstract of each article. You select only those articles of most interest to you for further reading. 